\title{
Conhecimento de enfermeiros acerca do cateter central de inserção periférica: realidade local e desafios globais
}

\author{
Nurses' knowledge of the peripherally inserted central catheter: local realities and global challenges \\ Conocimiento de los enfermeros acerca del catéter central de inserción periférica: realidad local y \\ desafíos globales
}

\author{
José Antônio de Sá Neto'; Aline Cerqueira Santos Santana da Silval'; Amanda Ribeiro Vidall'; \\ Virginia Maria de Azevedo Oliveira Knupp"v; Leila Leontina do Couto Barcia'; Ana Claudia Mateus Barretovl.
}

\begin{abstract}
RESUMO
Objetivo: analisar o conhecimento dos enfermeiros quanto à utilização do cateter central de inserção periférica (PICC) como dispositivo intravenoso na prática assistencial, em três hospitais públicos do Estado do Rio de Janeiro. Método: estudo descritivo seccional, através da análise univariada e bivariada, com cálculo das medidas de tendência central. Participaram do estudo 41 enfermeiros. Resultados: embora 38 (92,7\%) enfermeiros informaram conhecer o PICC, 29 (70,8\%) destes não possuíam habilitação para inserção do cateter; $23(56,1 \%)$ desconheciam as vantagens do dispositivo; $26(63,4 \%)$ informaram que o cateter não é indicado nas instituições pesquisadas, prevalecendo a punção profunda. Conclusão: a maioria dos enfermeiros pesquisados não possui o título de e habilitação para a indicação e inserção do referido cateter. Do mesmo modo, as instituições pesquisadas não indicam o PICC como dispositivo intravenoso, apesar de suas inegáveis vantagens, como a qualidade e a segurança na prática clínica.

Descritores: Cateterismo venoso periférico; capacitação; enfermagem; terapia intensiva.
\end{abstract}

\section{ABSTRACT}

Objective: to examine nurses' knowledge of use of the peripherally inserted central catheter (PICC), as an intravenous device in practical care, at three public hospitals in Rio de Janeiro State. Method: this descriptive, cross-sectional study used univariate and bivariate analysis and calculated central tendency measures. Results: although 38 (92.7\%) of the nurses reported knowing about the PICC, $29(70.8 \%)$ had no qualification in inserting the catheter; $23(56.1 \%)$ were unaware of the advantages of the device; and $26(63.4 \%)$ stated that the catheter was not indicated at the institutions studied, but that deep puncture predominated. Conclusion: most of the nurses surveyed lacked the title or qualification enabling them to indicate and insert the PICC. Likewise, the institutions surveyed did not indicate the catheter as an intravenous device, despite its undeniable advantages, such as quality and safety in clinical practice. Descriptors: Peripheral venous catheterization; training; nursing; intensive therapy.

\section{RESUMEN}

Objetivo: analizar el conocimiento de los enfermeros en cuanto a la utilización del catéter central de inserción periférica (PICC) como dispositivo intravenoso en la práctica asistencial en tres hospitales públicos del estado de Río de Janeiro. Método: estudio descriptivo seccional, a través del análisis univariado y bivariado, con cálculo de las medidas de tendencia central. Participaron en el estudio 41 enfermeros. Resultados: aunque 38 (92,7\%) enfermeros informaron conocer el PICC, 29 (70,8\%) entre ellos no poseían habilitación para inserción del catéter; $23(56,1 \%)$ desconocían las ventajas del dispositivo; $26(63,4 \%)$ informaron que el catéter no está indicado en las instituciones investigadas, que dan preferencia a la punción profunda. Conclusión: gran parte de los enfermeros encuestados no posee el título de habilitación para la indicación y la inserción del PICC. De igual modo, las instituciones investigadas no indican el PICC como dispositivo intravenoso, a pesar de sus innegables ventajas como la calidad y la seguridad en la práctica clínica. Descriptores: Cateterismo venoso periférico; capacitación; enfermería; terapia intensiva.

\section{INTRODUÇÃO}

A crescente complexidade imposta pelos processos de produção dos cuidados em enfermagem exige a reestruturação do modelo de gestão aplicado nas unidades de atendimento a pacientes com média e alta complexidade, visando atender com segurança e qualidade as reais necessidades apresentadas ${ }^{1}$.
Nesta vertente, a admissão do paciente na unidade de terapia intensiva (UTI) impõe grande desafio para equipe de saúde, que desempenha nesse contexto uma assistência integralizada ao paciente por considerar desde o estado catabólico da doença, alterações hemodinâmicas até o pronto restabelecimento clínico,

Enfermeiro. Mestre. Professor Assistente. Faculdade de Enfermagem da Universidade do Estado do Rio de Janeiro. Brasil. E-mail: jas.neto2009@gmail.com "Enfermeira Doutora. Professora Adjunta. Universidade Federal Fluminense do Campus Universitário de Rio das Ostras. Brasil. E-mail: alinecer2014@gmail.com "'Enfermeira, Graduada em Enfermagem pela Universidade Federal Fluminense do Campus Universitário de Rio das Ostras. Brasil. E-mail: amandarvidal@yahoo.com.br IvEnfermeira. Doutora. Professora Adjunta. Universidade Federal Fluminense do Campus Universitário de Rio das Ostras, RJ. Brasil. E-mail: virgulaknupp@yahoo.com.br vEnfermeira. Doutora. Professora Adjunta. Universidade Federal Fluminense do Campus Universitário de Rio das Ostras, RJ. Brasil. E-mail: leila_leontina@hotmail.com v'Enfermeira. Doutora. Professora Adjunta. Universidade Federal Fluminense do Campus Universitário de Rio das Ostras, RJ. Brasil. E-mail: amateusbarreto@gmail.com 
físico e psíquico tornando-se imperativo a prestação de uma assistência livre de danos².

A clientela referenciada para UTI apresenta-se em estado clinico instável, necessitando de intervenções imediatas e prolongadas, incluindo a necessidade de um acesso venoso duradouro e seguro, que permita a infusão de fluxos maiores e constantes de líquidos, como é o caso da nutrição parenteral total (NPT), infusão de drogas vasoativas, hidratação venosa com alta taxa de infusão de glicose (TIG) e fármacos variados ${ }^{3,4}$.

Com o aperfeiçoamento tecnológico e terapêutico, a utilização do peripherally inserted central cateter (PICC) tornou-se um recurso indispensável no tratamento de doenças agudas e crônicas, sendo um dispositivo central de primeira escolha, utilizado com diversas finalidades e situações variadas ${ }^{5,6}$.

Trata-se, de um dispositivo intravenoso seguro, inserido em vasos dispostos perifericamente, com localização central, devendo sua ponta ficar localizada em veias de grande calibre, como a cava superior e inferior, possibilitando a infusão de fármacos variados, soluções hiperosmolares, vesicantes/irritantes e terapias de longa duração ${ }^{7,8}$.

Para que haja sucesso na prática clínica com o PICC alguns fatores devem ser considerados, como a habilidade e capacitação técnica do profissional enfermeiro, conhecimento sobre o cateter, indicação, contraindicação, eleição do vaso que será puncionado, técnica de inserção, complicações, bem como, cuidados específicos para inserção, manutenção e remoção ao final da indicação terapêutica ${ }^{3}$.

Considerando o enfermeiro como o profissional majoritariamente responsável pela indicação, inserção, manutenção e retirada do PICC, cumpre destacar a seguinte questão: qual conhecimento o enfermeiro possui sobre esse dispositivo intravenoso?

Este estudo tem como objetivo analisar o conhecimento dos enfermeiros quanto à utilização do PICC na prática assistencial, em três hospitais públicos da região litorânea do Estado do Rio de Janeiro.

\section{REVISÃO DE LITERATURA}

Na atualidade, $90 \%$ dos pacientes hospitalizados recebem tratamento através da terapia intravenosa, tornando-se um dos procedimentos invasivos mais realizados no ambiente hospitalar, sendo uma prática majoritariamente desenvolvida pelo enfermeiro ${ }^{9}$.

Estudos tem demonstrado muitos benefícios através da prática clínica com o uso do cateter PICC (Peripherally Inserted Central Cateter) ou CCIP (Cateter Central de Inserção Periférica) Dentre os principais benefícios, destacam-se: menor risco de infecção, preservação da rede venosa, inserção menos traumática, maior tempo de permanência, redução de custos, menor risco de complicações, administração de soluções vesicantes/ irritantes, como antimicrobianos e drogas vasoativas, além da inserção segura à beira leito realizado por enfermeiros ${ }^{4,10}$. Fármacos, quando administrados por intermédio desse dispositivo se diluem mais rapidamente, diminuindo possíveis eventos adversos, somado ao menor custo e baixa taxa de infecção quando comparado ao Cateter Venoso Central (CVC) tunelizado ou cirurgicamente inserido ${ }^{10,11,12}$.

Para se obter melhores resultados, além do conhecimento técnico-científico, habilidade técnica, qualificação e capacitação para a inserção do cateter, o enfermeiro deve reconhecer os potencias riscos e complicações do uso do dispositivo, promovendo uma assistência qualificada e segura ao paciente ${ }^{13,14}$.

Nessa perspectiva, boas práticas em saúde, por meio do cuidado de enfermagem apresentam-se como ponto chave não só para a transformação, mas como também para a evolução científica. A terapia intravenosa é um dos procedimentos que demanda inovações na prática clínica pelo enfermeiro, fundamentada em bases científicas, como forma de obter melhores resultados assistenciais e indicadores de qualidade ${ }^{5}$.

\section{Metodologia}

Trata-se de um estudo descritivo seccional. A pesquisa foi realizada em três unidades hospitalares pública da regional de saúde Baixada Litorânea - Rio de Janeiro- RJ, Brasil. As unidades hospitalares foram elencadas primeiramente por oferecerem serviço de pediatria, neonatologia e unidades de atendimento ao adulto/idoso a pacientes mais vulneráveis, hemodinamicamente instáveis e mais, especificamente, suscetiveis à constante tentativa de punção venosa, o que possibilitou a investigação acerca do conhecimento e utilização do PICC - dispositivo intravenoso - por enfermeiros.

Atendendo aos aspectos éticos previstos na Resolução no 466/2012, os participantes foram enfermeiros que mediante apresentação do Termo de Consentimento Livre e Esclarecido (TCLE) aceitaram participar do estudo. Foram adotados como critérios de inclusão - ser e atuar como enfermeiros, lotados nos setores de unidade pediátrica, neonatal, unidades de atendimento ao adulto / idoso, geralmente, pertencentes à unidade de tratamento intensivo e/ou clínica médica, por no mínimo 3 meses, independentemente do tempo de formação em enfermagem. Foram excluídos do estudo outros profissionais de saúde que não fossem enfermeiros, tempo de atuação inferior a 3 meses e que não atuassem nos setores anteriormente citados.

A amostra por conveniência foi definida, considerando a totalidade de profissionais por setor, a aplicação dos critérios de inclusão e de exclusão, e a disponibilidade dos profissionais. Participaram do estudo 41 enfermeiros.

O projeto de pesquisa foi submetido ao Comitê de Ética em Pesquisa (CEP) da Universidade Federal Fluminense (UFF) e obteve aprovação sob o parecer número 1.220.205. 
A coleta de dados ocorreu entre novembro de 2015 a março de 2016. O instrumento de coleta de dados foi um questionário estruturado, constituído de duas partes, sendo a primeira parte destinada aos dados da caracterização dos participantes, relacionados à idade, sexo, tempo de formado, tempo em que trabalha na instituição, formação profissional como, curso de pós-graduação lato e/ ou strito sensu. A segunda parte foi relacionada aos aspectos diretamente envolvidos com o conhecimento e a prática clínica do PICC, entre eles: capacitação ou habilitação; onde o curso foi realizado, indicações do dispositivo, vantagens, profissionais autorizados a implantar, existência de normas, rotinas, Procedimento Operacional Padrão (POP), uso de protocolo para inserção, manutenção e retirada do PICC, entre outras.

Os dados coletados foram submetidos à análise univariada e bivariada, com cálculo das medidas de tendência central. Os dados foram tabulados no programa estatístico $R$ (https:www.r-project.org), que é de domínio público, cujos resultados são apresentados em tabelas.

\section{RESUltados e Discussão}

Participaram do presente estudo 41 enfermeiros que atuavam nas três unidades em diferentes turnos, dos quais 32 (78\%) eram predominantemente do sexo feminino. A média de idade foi de 35 anos. Dentre os participantes pesquisados, $35(85,4 \%)$ possuíam pósgraduação Latu Sensu e 3 (7,3\%) tinham como titulação maior o curso de mestrado. Destes, 16 (39\%) tem especialização na área de terapia intensiva adulta, seguida da especialização em terapia intensiva neonatal e pediátrica com $10(24,4 \%)$, e enfermagem do trabalho com 7 $(17,1 \%)$. Foi evidenciado um perfil de enfermeiros com tempo médio de profissão entre 5 a 10 anos. Quanto ao setor de atuação, a grande maioria, 11 (26,8\%) profissionais atuam na UTI, $11(26,8 \%)$ na enfermaria de pediatria e $6(14,6 \%)$ no setor de clínica médica.

Quando investigados se conheciam esse tipo de dispositivo, $38(92,7 \%)$ responderam que sim, e quando questionados onde obtiveram conhecimento sobre esse dispositivo, $18(43,9 \%)$ responderam que foi em universidades, seguido de 9 (21,9\%) durante a realização de cursos, $7(17,1 \%)$ em palestras e $4(9,7 \%)$ em serviço.

Apesar de um grande percentual entre os pesquisados possuir como título a especialização, dos 41 profissionais, 29 (70,8\%) destes não possuíam habilitação para inserção do PICC. Em relação aos profissionais habilitados, quando questionados onde foi realizado o curso de habilitação com o PICC, 4 (9,8\%) relataram ter realizado em hospitais, $6(14,6 \%)$ em universidades e $2(4,9 \%)$ em sociedades especializadas. Quanto a certificação, 1 (2,4\%) participante informou que obteve o certificado atendendo a exigência do curso em comprovar a inserção de um cateter, 3 (7,3\%) através da inserção de três ou mais cateteres e 8 (19,5\%) não tinham a comprovação de inserção. Constatou-se também, que 26 (63.4\%) enfermeiros das unidades hospitalares pesquisadas, não indicam o cateter como dispositivo intravenoso para a recuperação dos pacientes, conforme mostra a Tabela 1.

Sobre o conhecimento acerca do cateter, foi possível observar que, a maioria dos participantes alega conhecer o cateter. Entretanto, dentre os mesmos a maior parcela, apesar de possuir título de pós-graduação latu sensu e atuar em UTI, habilitada para a prática com o PICC.

Nota-se que a maioria dos enfermeiros não foi qualificada por sociedades especialistas e que também obteve o certificado de habilitação sem a comprovação prática de inserção de cateteres. Nesse sentido, percebe-se que não há uma determinação legal sobre a exigência de prática da inserção de cateteres por parte dos órgãos competentes e regulamentadores da profissão, ficando dessa maneira, a cargo das próprias entidades que oferecem o curso determinarem ou não a exigência de implantação de dois ou mais cateteres para se obter a certificação.

Apesar de seus benefícios, a prática clínica com o PICC envolve uma gama de fatores, que demanda conhecimentos tecnicos-cientificos específicos, e, portanto, capacitação e habilitação, com prioridade para a prevenção e a detecção precoce de complicações relacionadas ao uso do dispositivo, visando à segurança e ao bem-estar do paciente ${ }^{12}$.

Entre os fatores envolvidos nessa vertente, há de se destacar que o profissional, para aprofundar seus conhecimentos teórico e prático deve submeter-se a

TABELA 1: Conhecimento dos enfermeiros acerca do PICC e da habilitação para sua inserção. Três unidades pesquisadas, Baixada Litorânea - RJ, 2016

\begin{tabular}{|c|c|c|}
\hline Variáveis & $\mathbf{n}$ & $\%$ \\
\hline \multicolumn{3}{|l|}{ Conhece o PICC } \\
\hline Sim & 38 & 92,7 \\
\hline Não & 3 & 7,3 \\
\hline \multicolumn{3}{|l|}{ Como conheceu o PICC } \\
\hline Universidade & 18 & 43,9 \\
\hline Cursos & 9 & 21,9 \\
\hline Palestra & 7 & 17,1 \\
\hline Serviço & 4 & 9,7 \\
\hline \multicolumn{3}{|l|}{ Habilitado } \\
\hline Sim & 12 & 29,2 \\
\hline Não & 29 & 70,8 \\
\hline \multicolumn{3}{|l|}{ Onde realizou o curso } \\
\hline Hospital & 4 & 9,8 \\
\hline Sociedades & 2 & 4,9 \\
\hline Universidade & 6 & 14,6 \\
\hline Não informado & 29 & 70,7 \\
\hline \multicolumn{3}{|l|}{ Conferido certificado } \\
\hline Implantação de 1 & 1 & 2,4 \\
\hline Implantação de 3 ou mais & 3 & 7,3 \\
\hline Sem comprovação & 8 & 19,5 \\
\hline Não habilitados & 29 & 70,8 \\
\hline \multicolumn{3}{|l|}{ PICC é indicado na instituição } \\
\hline Sim & 15 & 36,6 \\
\hline Não & 26 & 63,4 \\
\hline
\end{tabular}


um curso de capacitação e habilitação, que é oferecido pela Sociedade Brasileira de Especialistas em Pediatria (SOBEP) e também pela Sociedade Brasileira de Enfermeiros em Terapia Intensiva (SOBETI), seguindo as diretrizes determinadas pela Infusion Nurses Society (INS) e pela Center For Diseases Control and Prevention (CDC), sediados nos Estados Unidos da América ${ }^{15,16}$.

Nessa diretiva, ressalta-se também a necessidade de inclusão desse conteúdo em curso de pós-graduação, assumindo um caráter mais prático na formação acadêmica, a fim de capacitar novos enfermeiros para a inserção do PICC. Assim, a qualificação e habilitação ofericidas por sociedades ou cursos devidamente legalizados apontam para uma prática mais segura e com menor risco ${ }^{17}$.

Quanto ao conhecimento sobre a indicação do PICC nas instituições pesquisadas, 8 (19,5\%) enfermeiros obtiveram um nível de acerto relacionado à infusão de soluções vesicantes/irritantes, como drogas vasoativas, hidratação venosa (HV), antibioticoterapia (ATB) e nutrição parenteral total (NPT); outro $1(2,4 \%)$, ressaltou-se seu uso no caso do esquema de antibiótico exigir tempo prolongado, geralmente superior a 7 dias; a maioria, 32 (78,1\%), não indicou o dispositivo na UTI adulta, segundo os resultados da Tabela 2.

Com relação à indicação do dispositivo, na condução da assistência prestada, foi possível observar que a maioria dos profissionais não indica o cateter como dispositivo de escolha. Tal fato está diretamente relacionado à falta de capacitação dos enfermeiros, e,

TABELA 2: Conhecimentos gerais dos enfermeiros acerca da prática clínica com o PICC nas três unidades pesquisadas. Baixa Litorânea- RJ, 2016

\begin{tabular}{|c|c|c|}
\hline Variáveis & $\mathbf{n}$ & $\%$ \\
\hline \multicolumn{3}{|l|}{ Indicação } \\
\hline Antibioticoterapia prolongada & 1 & 2,4 \\
\hline Drogas vasoativas, HV, ATB, NPT & 8 & 19,5 \\
\hline Não é indicado UTI adulta & 32 & 78,1 \\
\hline \multicolumn{3}{|l|}{ Vantagens } \\
\hline Controle hemodinâmico & 2 & 4,9 \\
\hline Diminuição das punções & 8 & 19,5 \\
\hline Segurança do paciente & 2 & 4,9 \\
\hline Tempo prolongado & 6 & 14,6 \\
\hline Desconhece & 23 & 56,1 \\
\hline \multicolumn{3}{|l|}{ Média de cateter/mês } \\
\hline 0 a 5 & 12 & 29,3 \\
\hline 5 a 10 & 9 & 21,9 \\
\hline Acima de 10 & 20 & 48,8 \\
\hline \multicolumn{3}{|l|}{ Profissional que implanta o PICC } \\
\hline Enfermeiro & 8 & 19,5 \\
\hline Médico & 1 & 2,4 \\
\hline Não implanta & 32 & 78,1 \\
\hline \multicolumn{3}{|l|}{ Protocolo/ POP/ Normas } \\
\hline Não & 32 & 78,1 \\
\hline Sim & 9 & 21,9 \\
\hline \multicolumn{3}{|l|}{ Limites e possibilidades/ PICC } \\
\hline Custo & 10 & 24,4 \\
\hline $\begin{array}{l}\text { Custo, desconhecimento e ausência de } \\
\text { profissionais capacitados }\end{array}$ & 31 & 75,6 \\
\hline
\end{tabular}

portanto, o desconhecimento deles acerca do uso desse dispositivo em pacientes de alta complexidade.

Referente às vantagens, foi constatado que 23 $(56,1 \%)$ enfermeiros entrevistados desconhecem as vantagens que o dispositivo intravenoso oferece; 8 (19,5\%) apontaram como principal vantagem a diminuição de punções venosas e $6(14,6 \%$,) indicaram a utilização do dispositivo na infusão por tempo prolongado. Quando questionados sobre a média de cateteres indicados por mês nas respectivas instituições, 20 (48,8\%) participantes relataram ser acima de 10 cateteres, 12 (29,3\%) informaram ser no máximo 5 cateteres e $9(21,9 \%)$ - de 5 a 10 cateteres.

Referente à média de cateteres que são indicados por mês nas instituições pesquisadas, a maioria alegou ser a média acima de 10 cateteres por mês, entretanto foi possível observar que em quase todas as instituições estudadas o cateter não é inserido.

Nesse sentido, torna-se imprescindível o incentivo do uso do cateter nas unidades hospitalares, sabendo que a não visibilidade do cateter é uma situação preocupante, e foi observado que isso se dá tanto devido ao custo quanto a capacitação de profissionais para o manuseio desse dispositivo, embora, seus benefícios sejam incontestáveis ${ }^{17,18}$.

Corroborando o fato, de a grande maioria dos participantes não ser habilitada para a prática com o PICC e não indicar o dispositivo, também a maioria - 23 $(56,1 \%)$ - alegou desconhecimento das vantagens da utilização desse recurso, conforme a Tabela 2.

Em relação ao profissional que implanta o cateter, $32(78,1 \%)$ dos entrevistados relataram não implantar, sendo que $8(19,5 \%)$ foram implantados por enfermeiros e $1(2,4 \%)$ por médicos. Dentre os entrevistados, 32 $(78,1 \%)$ responderam não existir em suas unidades de trabalho protocolos, normas, rotinas e/ ou POP como ferramenta administrativa na condução da prática clínica com o cateter, enquanto 9 (21,9\%) relataram a sua existência na instituição onde trabalham.

No Brasil, a realização desse procedimento está legalmente respaldada na Resolução no 258/2001, do Conselho Federal de Enfermagem (COFEN), em seu artigo 1 , a qual considera licito ao enfermeiro a inserção do cateter, desde que, conforme descrito no artigo 2 , esse profissional esteja devidamente qualificado através de curso regulamentado ${ }^{19}$.

No que concerne à observação de normas, protocolos e diretrizes, como ferramenta administrativa na condução da prática com o CCIP, a grande maioria dos participantes afirmou não utilizá-los. E dentre os limites e possibilidades para a utilização do cateter, a maioria - 31 (75,6\%) - apontou o custo, o desconhecimento e a ausência de profissionais capacitados, enquanto a minoria 10 (24,4\%) - apontou apenas o custo como fator limitante para a sua aplicação, conforme a Tabela 2.

O enfermeiro é o protagonista em muitas ações no seu cotidiano. No caso da prática com o PICC, ele 
é o responsável desde a inserção, manutenção até a sua retirada. Contudo, esse conceito deve extrapolar a valorização dos procedimentos apenas como técnica e abarcar os diversos conhecimentos técnico-científicos que sustentam essa prática. Como é o caso da existência e utilização de protocolos, normas, rotinas, POP, bem como, o Termo de Consentimento Informado ( $\mathrm{TCl}$ ) que direciona a prática de enfermagem no emprego desse cateter, visando normatizar um procedimento prescrito, o que torna fundamental para o seu êxito ${ }^{4,7}$

No que se refere aos limites e possibilidades do uso do PICC, uma pesquisa realizada no município de São Paulo comprovou que há uma diferença estatisticamente significativa sobre a predominância do uso do PICC pelas instituições privadas, podendo indicar que provavelmente o fator econômico dificulte sua incorporação à prática assistencial ${ }^{18}$.

Um estudo sobre esse tema fez uma comparação em relação ao custo do cateter, em que uma punção venosa periférica custa US\$ 32,00 e a inserção do PICC em torno de US\$200,00, o que aparentemente parecer ser mais caro, porém, quando considerado o tempo em que clientes internados na UTI necessitam de uma terapia intravenosa, geralmente superior há sete dias, o custo final do PICC é igual a de uma punção periférica ${ }^{20}$

Ainda, uma criança com acesso venoso difícil pode ser submetida, em média, de 12 a 20 tentativas de punção, no período de quatro semanas de internação, mostrando que, sob o ponto de vista financeiro, o uso do cateter pode ser uma alternativa mais que viável ${ }^{18}$

A respeito do dispositivo mais utilizado em paciente grave, a maioria dos profissionais relatou ser a punção profunda seu dispositivo de escolha, seguido do jelco utilizado na condução terapêutica do paciente, evidenciando total desconhecimento acerca das vantagens e segurança proporcionada pelo PICC.

Quando questionados sobre qual dispositivo é implantado no lugar do PICC, 20 (48.8\%) enfermeiros declararam ser o jelco em maior proporção, seguida da punção venosa profunda com 18 (43,9\%); assim, o manejo do paciente grave, com relação à terapia intravenosa, dá-se, sobretudo, através do jelco e da punção profunda, como mostra a Tabela 3.

TABELA 3: Conhecimento sobre dispositivo intravenoso mais utilizado em paciente grave e dispositivo implantado no lugar do PICC. Três unidades pesquisadas, Baixa Litorânea- RJ, 2016

\begin{tabular}{lcc}
\hline Variáveis & $\mathbf{n}$ & $\mathbf{\%}$ \\
\hline Dispositivo mais utilizado em paciente grave & & \\
Jelco & 9 & 22,0 \\
PICC & 8 & 19,5 \\
$\quad$ Punção profunda & 24 & 58,5 \\
Dispositivo implantado no lugar do PICC & & \\
$\quad$ Dissecção venosa & 3 & 7,3 \\
$\quad$ Jelco & 20 & 48,8 \\
Punção profunda & 18 & 43,9 \\
\hline
\end{tabular}

Uma pesquisa, realizada no Instituto Fernandes Figueira, mostrou que o número de cateteres inseridos no período de estudo e o número de efeitos adversos de causa infecciosa estão relacionados a uma taxa de infecção maior nos cateteres inseridos por via venosa profunda (34,2\%) seguida da dissecção venosa (26,3\%), em comparação com o PICC que apresentou uma taxa de $18,6 \%{ }^{19}$.

Estudos evidenciam que a punção profunda não deve ser a primeira escolha de acesso, em se tratando de paciente no limite de sua capacidade clínica e física. Quanto ao uso do jelco em pacientes vulneráveis, alguns riscos tornam-se eminentes, podendo levar à interrupção momentânea da terapêutica implementada e ao desenvolvimento de iatrogenias, como flebite, vasoespasmo, dor e infecção, além do aumento da permanência do paciente na unidade hospitalar ${ }^{21}$.

\section{CONCLUSÃO}

Considerando as experiências dos enfermeiros vivenciadas com o PICC e as inegáveis vantagens oferecidas pelo dispositivo, sobretudo, na condução do paciente grave, percebe-se uma prática incipiente e pouco expressiva com o cateter, entre as unidades pesquisadas.

Conclui-se que a maioria dos enfermeiros entrevistados não possui o título de capacitação e/ou habilitação para a indicação e inserção do PICC, do mesmo modo que não indica o cateter como dispositivo intravenoso de escolha.

Os resultados apontam a necessidade da visibilidade do cateter por parte dos profissionais, que na sua maioria desconhece a importância e as vantagens oferecidas por esse dispositivo na recuperação do paciente, bem como indicam a falta de investimento da fonte mantenedora das unidades hospitalares da região no referido dispositivo, interferindo no processo de cuidar, na recuperação clínica e qualidade de vida do paciente.

Apesar de ter alcançado o seu objetivo, este estudo possui limitações quanto à realização em apenas três unidades públicas hospitalares, com reduzida amostra, o que impede a generalização dos achados.

Esta pesquisa possui como potencialidade o retrato feito sobre a prática clínica com o cateter nessas unidades públicas. Todavia, indica a necessidade de novos estudos nacionais na enfermagem a fim de elucidar os questionamentos e controvérsias a respeito da prática clínica com o PICC.

\section{REFERÊNCIAS:}

1. Klock P, Erdmann AL. Caring for newborns in a NICU: dealing with the fragility of living/ surviving in the light of complexity. Rev Esc Enferm USP. 2012;46(1):45-51.

2. Melão MRC, Marcatto M. Humanização no cuidado neonatal: a concepção da equipe de enfermagem. Rev. pesqui. cuid. fundam. (Online). 2014;6(4):1409-18.

3. Oliveira CR, Neve ET, Rodrigues EC, Zamberlan KC, Silveira A. Cateter central de inserção periférica em pediatria e neonatologia: possibilidades de sistematização em hospital universitário. Esc. 
Anna Nery Rev. Enferm. 2014;18(3):379-85.

4. Reis AT, Santos SB, Barreto JM, Gomes GRS. O uso do cateter epicutâneo na clientela neonatal de um hospital público estadual: estudo retrospectivo. Rev. enferm. UERJ. 2011; 19(4):592-7.

5. Avelar AFM, Peterline MAS, Pedreira MLG. Assertividade e tempo de permanência de cateteres intravenoso periféricos com inserção guiada por ultrassonografia em crianças e adolescentes. Rev Esc Enferm USP. 2013;47(3):539-46.

6. Jacinto AKL, Avelar AFM, Wilson AMMM, Pedreira MLG. Flebite associada a cateteres intravenosos periféricos em crianças: estudo de fatores predisponentes. Esc. Anna Nery Rev. Enferm. 2014;18(2):220-6.

7. Moura PF, Contim D, Amaral JB. Cateter central de inserção periférica: revisão bibliométrica. Rev. enferm. atenção saúde. 2013;2(1):106-15.

8. Costa LC, Paes GO. Applicability of nursing diagnosis as grants for indication of Central Catheter of Peripheral Insertion. Esc. Anna Nery Rev. Enferm. 2012; 16(4):649-56.

9. Rangel RJM, Castro DS, Primo CC, Zandonade E, Christoffel $\mathrm{MM}$, Amorim MHC. Cateter central de inserção periférica em neonato: revisão integrativa da literatura. Rev. pesqui. cuid. fundam. (Online). 2016; 8(4):5193-202.

10. Costa P, Kimura AF, Vizzotto MPS, Castro TE, West A, Dorea E. Prevalência e motivos de remoção não eletiva do cateter central de inserção periférica em neonatos. Rev. gaucha enferm. 2012; 33(3):126-33.

11. Reynolds GE, Tierney SB, Klein JM. Antibiotics before removal of percutaneously inserted central venous catheters reduces clinical sepsis in premature infants. J. Pediatr. Pharmacol. Ther. 2015; 20(3):203-9.

12. Oliveira DFL, Azevedo RCS, Gaiva MAM. Diretrizes para terapia intravenosa no idoso: pesquisa bibliográfica. Rev. pesqui. cuid. fundam. (Online). 2014; 6(1):86-100.

13. Oliveira FT, Machado KMF, Santos LPR, Queiroz NR, Silva J, Paes $\mathrm{G}$. A práxis de acadêmicos de enfermagem aplicada à terapia intravenosa: um estudo antes e depois. Rev. enferm. UERJ. 2014; 22(2):219-25.

14. Baggio MA, Bazzi FCS, Bilibio CAC. Cateter central de inserção periférica: descrição da utilização em UTI neonatal e pediátrica. Rev. gaucha enferm. 2010; 31(1):70-6.

15. Silva ACSS, Santos EI, Queiroz PT, Góes FGB. O papel do enfermeiro com o cateter central de inserção periférica: revisão integrativa. Enferm. Atual. 2017;82(4):71-8.

16. Lourenço AS, Ohara, CVS. Conhecimento dos enfermeiros sobre a técnica de inserção do cateter central de inserção periférica em recém-nascidos. Rev. latinoam. enferm. 2010; 18(2):189-95. 17. Camargo PP, Kimura AF, Toma E, Tsunechiro MA. Initial placement of the peripherally insert central catheter's tip in neonates. Rev. Esc. Enferm. USP. 2008; 42(4):723-8.

18. Gomes AVO, Nascimento MAL, Silva LR, Santana KCL. Efeitos adversos relacionados ao processo do cateterismo venoso central em unidade intensiva neonatal e pediátrica. Rev. eletrônica enferm. 2012;14(4):883-92

19. Vendramim P, Pedreira MLG, Peterlini MAS. Cateteres centrais de inserção periférica em crianças de hospitais do município de São Paulo. Rev. Gaucha enferm. 2007; 28(3):331-9.

20. Conselho Federal de Enfermagem. Resolução $n^{\circ} 258,12$ de julho de 2001: Dispõe sobre a inserção do cateter periférico central por enfermeiros. 2001 [citado em 20 maio 2017]. Disponível em http://www.cofen.gov.br/resoluo-cofen-2582001_4296.html.

21. Petry L, Rocha KT, Madalosso ARM, Carvalho RMA, Scariot M. Peripherally inserted central venous catheters: limits and possibilities. Rev. eletrônica enferm. 2012; 14(4):937-43. 